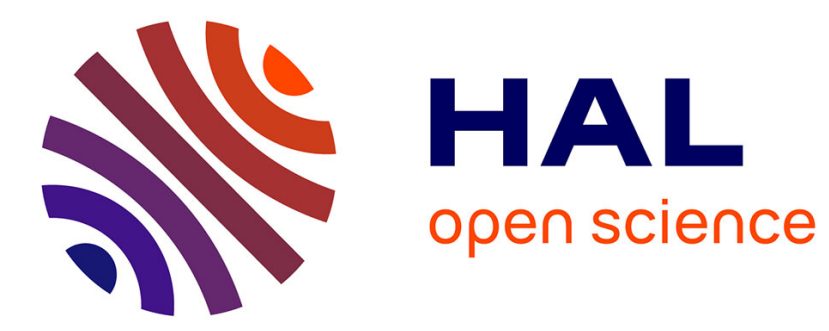

\title{
Éducation, Démocratie, Espérance
}

Denis Meuret

\section{To cite this version:}

Denis Meuret. Éducation, Démocratie, Espérance. Démocratie et éducation suivi de Expérience et éducation, Armand Colin, 22 p., 2011. halshs-00602115

\section{HAL Id: halshs-00602115 https://shs.hal.science/halshs-00602115}

Submitted on 21 Jun 2011

HAL is a multi-disciplinary open access archive for the deposit and dissemination of scientific research documents, whether they are published or not. The documents may come from teaching and research institutions in France or abroad, or from public or private research centers.
L'archive ouverte pluridisciplinaire HAL, est destinée au dépôt et à la diffusion de documents scientifiques de niveau recherche, publiés ou non, émanant des établissements d'enseignement et de recherche français ou étrangers, des laboratoires publics ou privés. 


\section{Éducation, Démocratie, Espérance}

Une introduction à l'édition 2011 de la traduction en français de deux ouvrages de John Dewey, Démocratie et Éducation et Expérience et Éducation_chez Armand Colin, Paris.

par Denis Meuret

(Université de Bourgogne (IREDU) et Institut universitaire de France)

L’école traverse aujourd'hui une passe étrange.

D’un côté, les économistes, les institutions internationales, certains politiques soutiennent que le développement de la scolarisation et l'amélioration de l'efficacité de l'école sont requis par l'émergente « économie de la connaissance ». Le capitalisme, après s’être longtemps méfié de l'école et n'en avoir attendu que des techniciens dociles et étroitement spécialisés, en attend aujourd'hui des travailleurs dotés de bonnes compétences générales, capables d’initiative ; il réclame une école efficace, et même équitable au sens restreint de capable de lui apporter des talents issus des catégories populaires. L’État attend de l'école croissance économique et cohésion sociale. Les parents et les élèves savent l'importance de la réussite scolaire pour trouver un emploi et sont d'autant plus mobilisés pour y atteindre.

De l'autre, l'école est la cible de critiques fondamentales, face auxquelles elle semble tétanisée, incapable de se justifier. Certaines soulignent que l'école française apparaît dans les comparaisons internationales comme très moyennement efficace et très sévèrement inéquitable (Baudelot et Establet, 2009). D’autres critiques portent au-delà de la situation française. Certains économistes pointent un coût croissant pour des résultats identiques, voire déclinants. Des psychologues trouvent paradoxal que l'école soit ennuyeuse alors que même les chimpanzés prennent plaisir à explorer le monde. Les intellectuels tiennent deux discours 
opposés. Pour les uns, l'école est devenue étrangère à la façon dont, aujourd'hui, les jeunes apprennent réellement. Dans le journal Le Monde (7 mars 2011), Serres juge que les réformes proposées aujourd’hui sont des « emplâtres diligentés selon des modèles depuis longtemps effacés » en sorte que l'école luit «d'un éclat semblable à celui des constellations dont les astronomes nous apprirent qu'elles étaient mortes depuis longtemps déjà». Divers indices, en effet, dont le développement du homeschooling, des leçons particulières et la difficulté de l'école à tirer parti de l’informatique, laissent penser que des formes individuelles d'apprentissage pourraient un jour la supplanter. Pour les autres, au contraire, il est encore temps de dissoudre le monde pour que survive l'école. La modernité rend l'enseignement plus difficile (Gauchet et al., 2008 ), il convient donc que l’école se raidisse contre la modernité. Luc Ferry, il n’y a pas si longtemps (2003), a prétendu gouverner l'école à partir d'un discours de ce type. Tous ces critiques partagent le diagnostic d'un écart considérable entre le monde et l'école ; ils divergent sur les solutions : les premiers en appellent à une adaptation de l'école aux nouvelles conditions de l'éducation, aux nouvelles formes de l'apprentissage ; les seconds avertissent qu'elle perd son âme à vouloir suivre une modernité qui mine ses valeurs, et en appellent à un sursaut républicain.

Il apparaîtra outrecuidant, un peu ridicule même, de prétendre que Démocratie et Éducation, et Expérience et Éducation, deux livres publiés respectivement en 1916 et en 1938 aux EtatsUnis, écrits par un auteur, John Dewey, qui vécut de 1859 à 1952, puissent aujourd'hui aider à construire un discours sur l'école capable d'orienter son gouvernement et de donner confiance et espoir à ceux qui la font tourner - et pourtant, elle tourne...- au quotidien. Imagine-t-on exhumer un traité de médecine des années trente pour éclairer les problèmes actuels de l'hôpital ? C'est pourtant bien ce que j'ai pensé, il y a quelques années, à la lecture de Démocratie et Éducation et de quelques autres ouvrages du même auteur. Je voudrais expliquer ici ce qui continue de me le faire penser. Pour cela, il faut d'abord prendre la mesure de la pensée de Dewey, ce qui n’est pas toujours facile, parce qu'elle est complexe, se déploie dans beaucoup de champs, et aussi, il faut bien le dire, qu’elle est écrite dans une langue contournée. Il faut ensuite insérer la conception de l'école de Dewey dans sa philosophie politique ; c’est en effet de l'actualité de la pensée politique de Dewey que découle pour l'essentiel l'intérêt de sa conception de l'école. C'est seulement ensuite que l'on pourra présenter cette conception, en nous appuyant sur les deux livres que nous introduisons ici, puis émettre quelques conjectures sur la fécondité de cette conception aujourd’hui. 


\section{Mesure de Dewey}

On peut distinguer deux phases dans la production intellectuelle de Dewey. La première phase est scientifique : de 1880 à 1920, il produit l'essentiel de son œuvre en psychologie et en philosophie, et aussi à propos de l'éducation. À bien des égards, Democracy and Education (1916), sa contribution la plus systématique à la conception d'une éducation démocratique, est le couronnement de cette période. L’importance de cette phase de la contribution de Dewey est illustrée par le beau livre de Louis Menand (2001). Menand retrace ce qu’il appelle la « refondation intellectuelle » des États-Unis après la guerre de Sécession. Il le fait à travers seulement quatre figures intellectuelles : Oliver Wendell-Holmes, qui a élaboré la doctrine juridique de la Cour Suprême, William James et Charles Pierce, les fondateurs du « pragmatisme »... et John Dewey, qui fut aussi un éminent représentant de cette philosophie. La seconde phase est plus politique : de 1920 à sa mort en 1952, Dewey publie surtout des textes courts, des interventions qui mobilisent son travail antérieur sur des problèmes d'actualité, y compris pour promouvoir la réforme de l'éducation mais aussi, dans Experience and Education (1938), pour traiter le problème créé par les dérives du mouvement de réforme de l'école qu'il a lui-même lancé. A propos cette fois de la contribution de Dewey à la première moitié du $\mathrm{XX}^{\mathrm{e}}$ siècle, on a pu écrire (aux États-Unis) qu'aucun débat ne pouvait être considéré comme clos tant que Dewey n’avait pas donné son opinion. Si on veut : un magistère politique et moral à la Victor Hugo, mais qui s’est exercé sur une période plus longue, un territoire plus vaste (Dewey est allé plaider pour une école démocratique au Japon et en Chine, il a présidé un « procès » de Trotski destiné à faire pièce à celui de Staline) et se fondait sur une philosophie au lieu d’être autorisé par une œuvre littéraire.

Après avoir dressé cette statue du commandeur, cependant, il nous faut aussitôt la détruire. L’influence de Dewey s'efface rapidement après la seconde guerre mondiale dans tous les domaines (le pragmatisme est démodé par la philosophie analytique, on rend ses théories sur l'école responsables du fait que les Soviétiques aient envoyé leur spoutnik dans l'espace avant les Américains, les marxistes lui reprochent sa naïveté quant à la nature de l’homme et d’ignorer la lutte des classes, etc.). Ses théories sont vilipendées - critiquées serait un euphémisme- par des intellectuels américains influents (Bloom, 1987), par le discours républicain sur l'éducation, pour ne rien dire bien sûr des sites créationnistes. Plus significatif peut être, Barack Obama, en 2009, a préféré nommer secrétaire à l’Éducation, non pas Linda Darling-Hammond, la fort deweyenne responsable de sa campagne présidentielle dans ce domaine, mais Arne Duncan, un partisan résolu de méthodes de gouvernement de l'école que 
les deweyens jugent « anathèmes » (Garrison \& Neiman, 2003) à l'égard de la pensée de Dewey.

Aujourd'hui, cependant, une troisième étape dans la réception de l’œuvre de Dewey semble s'amorcer, permise par un effacement de la philosophie analytique, à partir surtout d'une réévaluation de sa philosophie politique, aux États-Unis (Rorty, 1982/1993), comme en France (Zask, 2003 et dans cet ouvrage). Pour certains, ce retour en grâce vient de ce que l'époque est fatiguée du cynisme et accueille volontiers une pensée qui n’a pas honte d'être morale et de chercher à améliorer les choses. Pour d'autres, la philosophie politique de Dewey offre une possibilité de penser la démocratie de façon compatible avec la critique de la notion classique de l’individu. Des philosophes comme Hilary Putnam, Richard Bernstein, Richard Rorty «ont ramené vers Dewey la philosophie américaine contemporaine» (Festenstein, 2005). La philosophie de Dewey est aujourd'hui confrontée à celle de Deleuze (Semetzky, 2003), de Rawls (Weber, 2010, Weick,1993), de Foucault (Rorty, 1993, p. 369-370).

Cette description de la réception de l'œuvre de Dewey suggère qu'il convient d'éviter deux erreurs en abordant ce dernier. La première est évidente : Dewey n’est pas un simple « pédagogue ». La seconde l'est moins.

Les philosophes de l'éducation qui travaillent avec la pensée de Dewey, aux États-Unis (Gutman, 1999) ou en France (dont Gérard Deledalle, dont on lira en postface de cette édition un beau texte écrit pour la précédente), ne commettent pas l'erreur du pédagogue. Celle-ci est davantage le fait des partisans de tel ou tel type d'école, qui ont tendance à faire de Dewey un simple apôtre de la « pédagogie nouvelle », qu’ils y soient eux-mêmes plutôt favorables (cf. l'article « pédagogie nouvelle » du dernier Dictionnaire de l'Éducation [PUF, 2008]) ou qu'ils voient dans cette pédagogie l'amorce de la fin de la civilisation (ex. : Lurçat, 1999). Une autre façon de ne pas prendre la mesure de Dewey est de faire dériver sa conception de l'école d'un vague « progressisme » (Bulle, 2010). Une autre encore, paradoxale, est le fait de ceux qui, aux États-Unis, ont tellement l'habitude de penser l'école avec Dewey qu'ils finissent par penser Dewey à travers l'école. Par exemple, l’idée deweyenne que l'éducation est une fin en elle-même devient chez eux qu'il convient de laisser les enseignants libres de décider seuls de leur enseignement. Lorsque Rawls s’interroge sur ce que doit être l'éducation dans une société juste, il est accusé de commettre un crime de lèse-majesté deweyenne (Weber, 2010) parce qu'il subordonne l'éducation à un but externe. Les pédagogues (Freinet, Montessori, par exemple) sont le plus souvent très conscients des enjeux politiques et sociaux 
des pédagogies qu'ils proposent, mais Dewey fait le trajet inverse, il tire les conséquences sur l'école d'une philosophie qui couvre la philosophie de la connaissance, la psychologie et la philosophie politique.

Un fil court à travers ces trois domaines.

Philosophie de la connaissance. Dewey s’inscrit dans le courant « pragmatiste» (à distinguer bien sûr d'une position « pragmatique » : tout est bon pourvu que ça marche), dont on peut donner, peut être, une petite idée à travers deux maximes de son fondateur, Charles Peirce $(1878,1868)$ : « Notre conception d'un objet n’est rien d'autre que notre conception de ses effets», «le réel est ce qui résultera tôt ou tard de l’information et du raisonnement». La connaissance ne vient ni de l'intuition ni de l'introspection, mais seulement de notre relation au monde extérieur et de nos connaissances antérieures, de ce que Dewey appellera «expérience».

Psychologie. Dewey développe une psychologie de la connaissance. Dans un important article de jeunesse (« The reflex arc concept », 1896), il avance que même une activité réflexe ne doit pas être conçue comme une succession de stimuli et de réponses. En effet, nous appelons quelque chose stimulus seulement parce qu'il a été suivi d'une réponse. C’est en réalité à partir du tout que nous pouvons comprendre les parties, de la relation que nous pouvons comprendre ce qui la constitue, l'esprit n’est pas séparé du corps, c’est pourquoi c’est en faisant qu'on apprend. De même, l'effort n'est rien d'autre que « la tension entre les moyens et les fins et le sens de l'effort, la sensation de ce conflit » (« The Psychology of effort », 1897). Dewey élabore aussi une psychologie du développement : l'enfant n’est ni une cire sur laquelle l'éducation doit laisser son empreinte, ni un être dangereux qu'il faut civiliser, mais un individu en croissance, ce qu'il ne cessera d'être d'ailleurs à aucun moment de sa vie. Cela signifie qu'il n'est doté d'aucune autre nature que cette capacité à croître, ni une bonne qu'il suffirait de laisser s’épanouir, ni une mauvaise qu’il faudrait redresser.

Philosophie politique. Pour l'«ancien libéralisme» (celui de Locke, d’Adam Smith) les individus préexistent aux relations qu’ils nouent entre eux au sein de la société. Cette doctrine a permis de se débarrasser des formes absolues du pouvoir politique, elle a donc eu un rôle très positif, écrit Dewey, mais elle nuit aujourd'hui aux valeurs qu'elle servait : « la libération de l’intelligence, la liberté, la chance pour chaque individu de réaliser ses potentialités ». Elle est la principale justification du laissez-faire et par lui de la domination capitaliste. Cette menace contre la liberté est redoublée par le fait que, sur le terreau de cette société 
inégalitaire, prospèrent des idéologies encore plus dangereuses pour la liberté : communisme et fascisme. Le plus beau texte de Dewey sur cette question est peut-être Liberalism and Social Action (1938), écrit dans l'angoisse de la montée du fascisme en Europe. Il lui faut trouver la faille, ce qui ne va pas dans l'ancien libéralisme et dont il faut se débarrasser pour qu'il retrouve le pouvoir émancipateur qu'il a perdu et sur l'effacement duquel d'inquiétantes figures proposent leur service. La critique pragmatiste de l'ancien libéralisme lui indique cette faille. C’est précisément cette idée que les individus sont des entités indépendantes et en compétition les uns avec les autres. À l'inverse, affirment Dewey et le pragmatisme, les hommes ne sont des hommes que quand ils entrent en relation les uns avec les autres. Les individus émergent par un processus social, dans un dialogue critique et créatif avec le reste de la communauté. Dès lors, les institutions sociales ne sont pas des moyens pour obtenir quelque chose pour les individus, ce sont des moyens pour créer les individus. Dans une société démocratique, il n’y a pas opposition, mais renforcement entre l’amélioration de la société et l'approfondissement de l'individualité de chacun. Autrement dit, l'individu n'est pas au départ de la société, il en est, libre et divers, le but. L’alternative à la fois au laissezfaire et aux totalitarismes est un ensemble d'institutions qui ont pour but de créer des individus libres et divers, une diversité dont Dewey a retenu de Darwin qu'elle est la condition de la survie des sociétés.

On voit la cohérence de l'entreprise : le réel, les individus, les actions doivent être pensés à partir des relations qui les lient.

Toutefois, cette cohérence même se retourne contre Dewey de deux façons. D’une part, si adhérer au type d'école qu'il propose suppose que l'on adhère à tous les aspects de sa psychologie, de sa philosophie politique, au pragmatisme en général, il est possible que peu adhèrent. D’autre part, Dewey, surtout dans sa première période, semblable en cela à beaucoup d'auteurs de cette époque, développe une confiance qui paraît aujourd'hui excessive dans la capacité de la science à construire un monde nouveau (par exemple, la «nouvelle psychologie », celle de James, de Dewey, rendrait forcément caduque l’ancienne pédagogie). Il en est résulté - c’est la seconde erreur à ne pas commettre - que Dewey a été lu avec une révérence excessive, comme un «maître à penser », et aussi que ceux qu'il a inspiré ont cru un peu trop qu'ayant pour eux le progrès et la science, leur victoire était seulement une question de temps, les obstacles qu'ils rencontraient, des combats d'arrière-garde. Nous savons aujourd'hui que la « pédagogie traditionnelle » résiste bien trop pour qu’on puisse y voir seulement une survivance de la tradition. Autrement dit, nous savons que l'affaire est 
politique, ce qui, en termes deweyens, signifie qu'il nous faut montrer que Dewey nous aide en effet à concevoir des formes politiques, des institutions sociales (dont l'école), qui ont de meilleurs effets que celles qu’on leur oppose.

Cela incite à s’intéresser aux fondements politiques de l'école deweyenne, soit le thème fondamental de Démocratie et Éducation.

\section{Les fondements politiques de l'école démocratique}

Au fondement de la philosophie politique de Dewey, me semble-t-il, se trouvent deux idées. La première est l’infinie variété de la nature humaine. « L’idéal de la démocratie tient pour acquise l'infinie variété de la nature humaine et l'infinie pluralité des buts pour lesquels les hommes s’associent » (Social Absolutism, 1921/1983, cité par Weber, 2008). On trouve la seconde au début de Démocratie et Éducation, elle est banale à certains égards mais quiconque la lit après s’être un peu frotté à la philosophie politique du XVII et du XVIII siècle doit, me semble-t-il, en recevoir un choc. Non, dit Dewey, les hommes ne sont pas égoïstes, « même si des systèmes de gouvernement et des théories de l'État ont été construits sur cette notion ». Certes, il leur arrive de l'être, mais ce qui domine plutôt, c'est qu'ils sont enclins à coopérer. Le lecteur range alors Dewey du côté des philosophes pour qui l’État prolonge la société, garantit un ordre qui lui préexiste, John Locke, Adam Smith (qui n’est pas seulement un économiste), et non de l'autre côté, celui des philosophes pour qui seule l’existence de l'État permet à la société de fonctionner, soit qu'il empêche la guerre de tous contre tous en échange de l'obéissance (Hobbes), soit qu'il émane d’un sursaut, le Contrat Social de Rousseau, qui fait échapper la société à l'anomie, qui permet aux hommes de construire une société réconciliée avec la nature sur les ruines de la société corrompue du « second état de nature », qui est aussi, bien sûr, la société aristocratique.

Mais le lecteur note aussi, d'où le choc, que personne avant Dewey n’a donné une vision aussi générale, aussi généreuse de la propension des hommes à faire société, plus large que l'existence de droits antérieurs à l’État chez Locke, plus large que la « propension à échanger » de Smith. Mieux, Dewey donne un argument lumineux en faveur de cette propension à coopérer : « Sinon, aucune société n’existerait. » Croyez-vous, demande au fond Dewey, que la puissance de l’État suffirait à obliger à coopérer des hommes mus seulement par l'envie de se faire la guerre, ou de se faire valoir au détriment des autres ? Bien sûr, la 
guerre ; bien sûr, la volonté de se distinguer ; mais, si nous voulons comprendre que les sociétés existent, il nous faut accepter l'idée que ces dispositions négatives sont au fond moins puissantes que la propension à coopérer. Insistons : Dewey ne suppose pas un homme doté d'une bonne nature, soucieux de faire le bien, mû par l'altruisme. Dans nos deux ouvrages, il rompra des lances contre ceux qui supposent cette bonne nature chez l'enfant. Aucun besoin, pour suivre Dewey, de supposer que la coopération ne rencontre ni obstacles ni conflits, qu'elle ne peut s'interrompre. Il faut et il suffit, pour cela, d'accepter l'idée que, derrière ces conflits, ces obstacles, la propension à coopérer agît toujours dans l'autre sens et qu'il s’agit d'une tendance plus profonde que les autres.

On devine que tout ceci aura des conséquences importantes pour l'école : non pas une école qui nous fait accéder à la citoyenneté contre nos mauvais penchants, nos désirs immédiats, notre crédulité, nos idées fausses, mais une école qui favorisera la diversité des individus et leur donnera les outils de leur coopération. Mais revenons à la démocratie selon Dewey.

On peut distinguer trois niveaux de la démocratie chez Dewey (Festenstein, 2005) : Elle est un moyen de protéger les intérêts du peuple des décisions des experts et des puissants (démocratie électorale), elle est ensuite une façon de traiter les conflits d'intérêts qui surviennent dans une société, un forum et une méthode pour discuter et comprendre ce que sont nos intérêts et les caractéristiques des buts légitimes (démocratie participative, pourraiton dire). Mais elle est davantage, et c'est dans Démocratie et Éducation, que nous trouvons la formulation qui rend le mieux compte de la singularité de la conception deweyenne : « La démocratie est davantage qu'une forme de gouvernement. Elle est d'abord un mode de vie associé, d'expériences communes communiquées ${ }^{1}$.» La valeur d’un groupe social se mesure d'une part à la fréquence et à l'intensité des échanges entre ses membres, d'autre part à la fréquence des relations entre son intérieur et son extérieur. Inversement, une société indésirable est une société qui met des obstacles à l'accomplissement et à la communication libre des expériences. Ce que vise la démocratie et qui la soutient à la fois est « l'élargissement des intérêts partagés et la libération d’une plus grande diversité de capacités personnelles » (DE, p. $83^{2}$ ), d'où il suit que « la conscience claire de la vie commune dans

\footnotetext{
${ }^{1}$ Nous donnons ici la traduction qu'on trouvera dans cet ouvrage de « A democracy is more than a form of government ; it is primarily a mode of associated living, of conjoint communicated experience » (1916/2004, p. 83).

${ }^{2}$ Sauf indication contraire, la pagination de Démocratie et Éducation (DE) est celle de l'édition américaine de 1916, republiée en fac-similé par Dover (2004). Traduction, DM.
} 
toutes ses implications constitue l’idée de la démocratie » (Dewey, 1927, p. 156). L’ennemi de la démocratie est la peur, parce qu'elle isole, qu'elle empêche les échanges d’expériences, écrit Dewey bien avant les déboires des régimes totalitaires avec facebook et un peu avant que Roosevelt ne prononce sa célèbre phrase « La seule chose dont nous devons avoir peur, c’est la peur elle-même ».

De la diversité et de l'intensité des échanges naît le progrès. Ce dernier n'est pas une sorte de mécanisme externe, il est le produit des changements introduits par l'expérience et l'expérimentation dans une société démocratique qui a « le changement pour idéal » (DE p. 77). Dès lors, la délibération démocratique ne consiste pas à disputer de la conformité de l'action publique aux principes qui fondent l'État ou au sens de l'histoire, mais en une enquête qui permet au public de délibérer sur ses effets potentiels (Dewey, 1927). L’expérimentation est au cœur de la vie publique.

Cette conception de la démocratie a des conséquences importantes pour l’école.

Puisque la liberté s’éprouve dans l'expérience, il faut la concevoir comme la capacité d'agir librement, comme le pouvoir de faire, davantage que comme la capacité de penser librement, à l'écart de l'idéologie dominante, des croyances que les puissants veulent nous imposer (Condorcet). Dès lors, l’objectif de l'école sera, selon un mot difficile à traduire, l'empowerment des individus, l'accroissement de leur capacité à agir, davantage que leur dessillement, comme c’est le cas à partir des récits de Condorcet ou de Durkheim (Meuret, 2007).

De ce que Dewey définit l’individualité comme la capacité de choisir, de réfléchir sur ses buts, il suit que l'école doit développer ces capacités là. L’école doit produire des individus libres, imaginatifs, créatifs puisque c'est ce type d’individu qui sera le plus à même de favoriser la diversité et la richesse des expériences et des échanges qui elles-mêmes produiront des individus plus libres et plus riches. De ce que la société démocratique est une société « mobile, pleine de canaux par lesquels le changement peut arriver n'importe où », il suit que l'école « doit s’assurer que ses membres sont éduqués à l’adaptabilité et à la prise d'initiative personnelle » (DE, p. 84). Il y a une correspondance étroite entre une société démocratique qui « a le changement pour idéal » (DE, p. 77) et une éducation conçue comme un « moyen d’améliorer la société » (DE, p. 76). 
Quant aux contenus à enseigner, il en résulte l’importance des outils de communication (la langue au premier chef) et bien sûr des sciences, conçues non comme ce que nous savons des lois éternelles qui gouvernent le monde ou la nature, mais comme le résultat toujours provisoire d'expérimentations.

Par ailleurs, l’école ne doit pas être coupée du monde - une telle école serait, pour Dewey, « futile » -, et ceci dans deux dimensions, sociale et temporelle. Une expérience éducative présente évidemment des caractéristiques propres, mais elle n’a de sens que par les liens qu'elle entretient avec les différentes formes du social puisque son objectif est de permettre aux individus de vivre des expériences sociales plus complexes et plus riches. La responsabilité morale de l'école est de « permettre à l'enfant de comprendre et de respecter l'ensemble de ses relations sociales. Ce n'est pas seulement un futur citoyen, mais aussi un futur parent, un futur travailleur, un futur membre de la communauté » (DE, p. 95). L’école doit mimer le monde qui l'entoure. Ainsi, même à l'école, c'est du monde que l'élève apprend via le maître. Au point de vue temporel, il n’y a pas de coupure entre le moment où l'école essaye d’inculquer aux élèves une culture de haut niveau, des valeurs élevées et celui où la société tentera de les attirer vers tout le contraire, de fausses valeurs et une fausse culture. Au contraire, le propre d’une expérience éducative est qu'elle permet de mieux tirer profit, de mieux apprendre, d’expériences ultérieures, scolaires ou sociales. L’école doit donner à l'élève la capacité et l’envie de continuer à apprendre.

Il en résulte enfin une école équitable et laïque. Il est rare qu’on se soucie d’égalité des chances à l'époque où Dewey écrit. Mais, pour lui, la distinction qui prévaut dans l’école traditionnelle, entre une culture scolaire élevée pour l'élite et une formation étroite et spécialisée pour le peuple, doit être abandonnée. Elle n’est que le produit de la coupure sociale entre une classe de loisir et la classe laborieuse, et de la coupure métaphysique entre l'âme et le corps, deux coupures aussi contingentes l'une que l'autre. Inversement, « une société à laquelle la stratification en classes séparées serait fatale doit s’assurer que les chances intellectuelles sont accessibles à tous facilement et également » (DE, p. 84). L’équité selon Dewey ne se réduit pas à l'égalité des chances, elle implique en outre que tous atteignent un certain niveau de compétences, ce niveau de capacité d’initiative qui leur permettra « de n'être pas écrasés par les changements dans lesquels ils sont pris et dont ils ne percevraient pas la signification » (DE, p. 84), avec pour résultat que quelques uns s’approprieraient les produits de l'activité aveugle des autres. Dewey, sauf erreur, n'utilise pas le mot « laïcité ». Pourtant, à bien des égards son école apparaît plus laïque que l’école 
traditionnelle, en particulier l'école traditionnelle française, où le savoir est sacralisé et où l'excellence scolaire manifeste une capacité intellectuelle et morale qui est une forme de sainteté. Au contraire, la philosophie de l'expérience reste résolument à ras de notre terre. Rorty l’a bien vu : Dewey partage avec Foucault « un effort visant à libérer le genre humain de ce que Nietzsche appelait "le plus vieux mensonge”, celui qui nous persuade que, au-delà des expériences hasardeuses et périlleuses que nous accomplissons, il existe quelque chose (Dieu, la Science, la Connaissance, la Rationalité ou la Vérité) qui, si seulement nous observons les rituels qui conviennent ${ }^{3}$, nous permettra de franchir un pas dans la voie du Salut »(Rorty, 1993, p. 373).

\section{L'école selon Dewey}

Dewey construit sa conception de l'école à partir de sa philosophie politique et de sa psychologie. De la première, relève une série de caractéristiques des buts et de l'organisation sociale de l'école. De la seconde, relèvent plutôt les caractéristiques de l'enseignement, de la pédagogie, si l’on veut, dans la classe.

\section{L'enseignement, la pédagogie}

Puisque l'école ne vise pas à protéger la société des nouveaux venus mais à leur donner le pouvoir d'agir, puisque l’éducation repose sur l'expérience - « l'éducation est une reconstruction de l'expérience qui ajoute à la signification de l'expérience et qui augmente la capacité à diriger le cours des expériences ultérieures» (DE, p. 74), puisque Dewey place l'école dans la continuité des modes d'apprentissage informels qui l’ont précédée, l'élève apprend du monde, non de la parole du maître. Le maître doit organiser des situations d'apprentissage qui miment le monde ${ }^{4}$ et guider l'élève dans son apprentissage plutôt que délivrer un savoir ex cathedra. En ce sens, Dewey est en effet un partisan des « méthodes actives », du « learning by doing », même s’il a écrit Experience and Education pour

\footnotetext{
${ }^{3}$ L'entrée à Normale Sup, la sortie dans la botte de l'ENA, sont des exemples de ces rituels dans l'école française.

${ }^{4}$ Les « serious games », de plus en plus utilisés pour la formation professionnelle, des médecins ou des pilotes d'avion entre autres, utilisent les riches possibilités de l'informatique pour mimer des situations réelles à l'intention des étudiants.
} 
préciser que l'on n’apprend pas de n'importe quelle « activité ${ }^{5}$ », qu’il ne suffit pas de prendre le contrepied de l'éducation traditionnelle pour construire une éducation démocratique (EE, p. $20^{6}$ ). Que l'école vise à favoriser les échanges d'expériences milite pour le travail en groupe. « Le professeur quitte la position de patron ou de dictateur externe pour celle de guide des activités du groupe » (DE, p. 59).

Pour Dewey, l'enfant est défini par son immaturité, sa plasticité - sa capacité à tirer de l'expérience des facteurs qui modifieront son activité ultérieure, et donc à développer des dispositions nouvelles (DE, p. 44) -, ainsi que par sa capacité et son désir de croître (DE, p. 41), et de croître indéfiniment puisque la perfectibilité humaine est indéfinie. Sa nature, on l'a dit, n'est ni bonne ni mauvaise (en particulier, l'enfant n'est pas foncièrement égoïste), mais elle n’est pas « antisociale », comme Dewey reproche à Rousseau de le penser (DE, p. 87).

De cette plasticité découle la diversité des individus et de la façon dont ils apprennent, c'est donc la tâche de l'enseignant d'adapter son enseignement à chacun de ses élèves (DE, p. 162) de façon à rencontrer son intérêt, rencontrer et non susciter puisque Dewey se méfie des façons artificielles, externes de susciter l'intérêt - comme c'est le cas quand on pense que l'élève apprendra mieux avec un ordinateurs parce qu'il associe cette machine à des dimensions positives. Intéressé par une tâche qui fait sens pour lui, l'élève sera capable de persévérance, de la capacité de mener une tâche à son terme sans se laisser décourager par la difficulté ni distraire par l'environnement (DE, p. 123), persévérance qui est pour Dewey l'essence de la discipline scolaire, discipline qui se distingue de l'entêtement en ce qu'elle caractérise une activité dont l'élève a mûrement fait sien le but (DE, p. 124).

Une telle éducation a pour but d'apprendre à apprendre (DE, p. 44). Il ne s'agit pas de mettre en place des cours de technique d'apprentissage, mais de ce que l'éducation permet aux individus de croître si elle les rend capables de continuer à apprendre et si elle leur donne le désir de le faire. C'est en apprenant que l'on apprend à apprendre. C'est en ce sens qu'il faut comprendre l'idée que l'éducation est sa propre fin (apprendre de l'expérience permet de

\footnotetext{
${ }^{5}$ Interrogée sur ce qu'elle avait retenu de « l'enquête » organisée par son professeur d'histoire dans le camp militaire proche de son collège, une élève de $4^{\mathrm{e}}$ : «Que les militaires sont gentils ». Deux types d'activité, dit Dewey, sont anti-éducatives : les « activités de routine » et les « activités capricieuses » (DE, p. 74).

${ }^{6}$ La pagination des citations de Experience and Education renvoie à l'édition Touchstone books (New York, Simon and Schuster, 1997), traduction DM.
} 
vivre des expériences plus enrichissantes, dont on apprend davantage), ni dans le sens d'une éducation qui n'aurait aucun lien avec le monde profane, ni dans celui où l'enseignant devrait se soucier d'un développement de l'enfant qui n’aurait rien à voir avec son insertion sociale. Ce serait oublier que l'école est pour lui une agence d'amélioration de la société ou ce que Dewey écrit sur l'efficacité sociale (social efficiency) de l'éducation, à laquelle nous allons maintenant nous intéresser.

\section{Les buts et l'organisation de l'école}

Que Dewey ne soit pas un « pédagogue » se voit aussi à ce qu'il ne traite pas seulement de ce qui se passe en classe mais de l'ensemble des questions qui concernent le système scolaire, dont nous retiendrons ici : ses buts, l’articulation entre enseignement général et enseignement professionnel et la question de l'équité.

L'école démocratique, dit-il, articule trois buts alors que d'autres conceptions de l'école ont commis l'erreur de ne privilégier qu'un seul des trois : favoriser le développement naturel de l'enfant, transmettre la culture, servir l'utilité sociale (DE, chapitre 9). En particulier, l'éducation démocratique ne dédaigne pas l’objectif d’utiliser les « ressources économiques de façon utile » (DE, p. 114). Elle a un objectif « d'efficacité sociale ». Bien sûr, dit Dewey, il y a danger qu'en insistant trop exclusivement sur cet aspect on « accepte comme telles les conditions et normes économiques actuelles » (DE, p. 115) de même d'ailleurs qu’on les accepte aussi si l'on insiste trop exclusivement sur la transmission de la grande culture. Deux traits caractérisent le rapport de l'éducation démocratique à l'efficacité sociale : d'abord le fait que celle-ci « ne soit pas obtenue par une contrainte négative mais par l’usage positif des capacités naturelles dans des métiers qui ont une signification sociale » (DE, p. 114), ce que l’on pourrait formuler aujourd'hui, me semble-t-il, « une éducation démocratique doit attacher la plus grande importance à l’utilité économique et sociale de ses formations, mais, inversement, une économie démocratique doit être capable de rémunérer à leur juste valeur les compétences de ceux qui ont véritablement développé le mieux possible leurs capacités de faire, de sorte que chacun puisse être encouragé à le faire ». Ensuite, l'équité, mais d’une façon liée à ce qui précède. « La société industrielle, comme toutes celles qui l’ont précédée, est pleine d'iniquités. C’est le but de l'éducation progressive de contribuer à les corriger plutôt qu’à les perpétuer » (DE, p. 115). Pour cela, il faut refuser que l'acceptation du statuquo domine l'éducation professionnelle, soit, très concrètement, que les chances d'emploi dominent l'orientation des élèves, ce qui nous ramène à l'objectif précédent. Il en résulte que 
Dewey refuse de séparer radicalement enseignement professionnel et enseignement général. L’enseignement, pour lui, doit préparer à tous les métiers de la même façon. Il s’étonne que l'on regarde la formation pour devenir enseignant comme ressortissant à la culture tandis que celle qui conduit aux professions de mécanicien, de marchand, de médecin est considérée comme de la formation professionnelle. Il aurait pu ajouter, s’il avait traité du système français : que l'on regarde les études en lycée professionnel, mais non en école d’ingénieurs ou en médecine, comme de la formation professionnelle. Selon lui, es formations qui conduisent aux métiers subalternes doivent, elles aussi, permettre à ceux qui vont les exercer de « percevoir dans leur travail quotidien tout ce qu’il contient de signification générale et humaine ${ }^{7}$ » écrit-il dans The School and Society (1899). Il ajoute que l'enseignement traditionnel est moins « gratuit » qu'il n'est impuissant à améliorer l'humanité de quiconque d'autre que des clercs. L’objectif de l'école démocratique, en revanche, est qu'aucun travailleur ne soit un esclave au sens de Platon, soit « quelqu'un qui exprime les idées d'un autre par ses actions » (Dewey, 1899, p. 306).

Dewey ne se satisfait pas de la séparation du système d'enseignement en système primaire pour le peuple et système secondaire pour la bourgeoisie. Il observe, toujours dans The School and Society (1899), que $1 \%$ seulement de la population accède à l'enseignement supérieur et $5 \%$ au lycée et que plus de la moitié quitte l'école avant la fin de la cinquième année d'enseignement et il poursuit : « Tandis que les responsables de notre éducation nous parlent de grande culture, de développement de la personnalité, etc. comme de la fin et du but de l'éducation, la grande majorité de ceux qui fréquentent l'école la considèrent comme le moyen pratique d'ajouter un peu de pain et de beurre à une vie rétrécie » (Dewey, 1899, p. 309).

Quel rapport entre cette école et celle d'aujourd'hui, son fonctionnement actuel et les défis qu'elle doit affronter?

\section{Dewey et l'école aujourd'hui}

\footnotetext{
${ }^{7}$ L'héroïne du roman de Russel Banks, De beaux lendemains, conductrice de bus scolaire dans une région au climat difficile, a baptisé son bus d'un drôle de nom, ce qui le rend plus accueillant aux enfants. Dewey aurait trouvé, je crois, que cela montrait qu'elle avait perçu la « signification humaine » de son travail.
} 
L’actualité de Dewey peut être interrogée de plusieurs façons : dans quelle mesure le travail scientifique actuel sur l'apprentissage vérifie-t-il les théories de Dewey ? L’école aujourd’hui se rapproche-t-elle ou s’éloigne-t-elle de l'école démocratique ? Qu'a à proposer l'école démocratique face aux défis que rencontre l'école?

Décrire l’héritage scientifique de Dewey dépasse le cadre de cet exercice et les compétences de son auteur. Cependant, il semble qu'on puisse exclure l'idée que les théories de Dewey seraient démenties par les recherches empiriques contemporaines. Non pas que les travaux actuels valideraient la " pédagogie progressiste » au détriment de toute autre. Les recherches sur l'efficacité de l'enseignement indiquent, par exemple, comme la forme d'enseignement probablement la plus efficace un enseignement jugé hétérodoxe par les disciples de Dewey, l'enseignement direct et interactif, dans lequel le maître présente les contenus de façon frontale à la classe avant de diriger les élèves dans des travaux où ils les appliquent à la solution de problèmes. Cela n'invalide pas la pédagogie de projet, mais appelle sans doute à une réflexion sur l'articulation optimale entre des temps de transmission de savoirs-outils et des temps de mobilisation de ces outils dans des situations proches du réel. D’une façon générale, la psychologie expérimentale accorde une importance non anticipée par Dewey à la mémoire, en particulier à la mémoire à long terme, à la capacité d'automatiser des tâches pour pouvoir appliquer sa réflexion à des problèmes plus complexes. En revanche, les sciences cognitives confirment la diversité des modes d'apprentissage, le rythme variable de l'acquisition des capacités, la genèse des représentations à partir de l'action, trois points centraux chez Dewey.

Au-delà de sa théorie de l'apprentissage, deux aspects de l'école deweyenne semblent poser problème aujourd'hui. On verra dans les premiers chapitres de Démocratie et Éducation que Dewey inscrit l'école dans la continuité de l'éducation informelle que les enfants pouvaient recevoir dans les communautés étroites des sociétés traditionnelles, dans une économie artisanale, éducation que l'école doit en quelque sorte styliser. Dans son introduction à une précédente édition d’Expérience et Éducation, M.A. Carroi écrit : « Aussi est-il urgent de recréer un climat, celui de l'échoppe et celui de la ferme, où, naturellement, se multiplient les rapports actifs et vivants ". C'est bien ce qui pose problème, en ce que cela suggère que la société moderne ne présente aucune situation d'apprentissage dont l'école puisse s'inspirer, le risque étant que cet emprunt au passé ne fournisse des situations aussi artificielles et coupées du monde réel que l'éducation traditionnelle. Le second aspect n'est pas sans lien avec le premier. Dewey écrit que « ce que les parents les meilleurs et les plus sages veulent pour leurs 
enfants, la communauté doit le vouloir pour ses enfants »(Dewey, 1899). Amy Gutman (1999, p. 13-14) critique l'étroitesse et le flou de ce critère, qui laisse chacun libre de projeter ses propres idées sur celles des parents les plus sages, et trouve plus conforme à une éducation démocratique le fait que la façon d'éduquer les enfants soit le produit d'une délibération démocratique encadrée par deux principes, celui de la non répression des façons rationnelles de penser, même si elles ne sont pas populaires, et celui de la non discrimination entre les élèves.

Cela dit, ce qu'on observe est plutôt que les conceptions contemporaines de l'éducation se rapprochent de celle de Dewey. Cela se voit aux États-Unis, dans les institutions internationales, et même en France où pourtant un modèle alternatif et également très articulé d’éducation a été proposé par Durkheim (Meuret, 2007).

L’héritage américain de Dewey n'est sans doute pas là où ses adversaires le voient, dans cette éducation « romantique », " centrée sur l'enfant », dont il met tant de soin à se démarquer dans les deux ouvrages proposés ici au lecteur. Il réside dans des caractéristiques de l'école américaine qui sont moins controversées dans ce pays, qui y sont donc moins perçues, mais qui distinguent le système scolaire américain du français, par exemple : une conception positive, et non négative, de l’orientation des élèves ; la possibilité pour un élève de faire les meilleures études même si ses capacités ou son appétence pour le travail se révèlent tardivement ; l'existence d'un tronc commun jusqu'à la fin du secondaire ; la rareté des redoublements. À ces caractéristiques majeures, on ajoutera un détail. Dans le questionnaire adressé actuellement aux professeurs du district de New York pour recueillir leur évaluation de leur établissement scolaire, on leur demande leur opinion sur cette affirmation : " Mon école offre une assez grande variété d'activités et de cours pour susciter l'engagement des élèves ${ }^{8}$.» Outre que Dewey aurait sans doute trouvé bon qu'on demande aux élèves, à leurs parents et aux enseignants leur opinion sur leur établissement, l'idée que chaque élève doive pouvoir choisir ce qui suscite son intérêt est fort deweyenne. Il est probable que les concepteurs de ce questionnaire n’ont pas lu Dewey. Ce détail ne mesure donc pas « l’influence » directe de Dewey, mais une influence indirecte, à travers un récit qui a marqué et marque encore l'esprit public américain à propos de l'éducation, récit qui justifie entre autres le foisonnement des options dans les lycées américains.

\footnotetext{
${ }^{8}$ Les questionnaires de tous les établissements sont consultables sur le site du New York City Department of Education/schools in the community/Performance and accountability/ NYC data/NYC school survey.
} 
Cependant, dans les années 1980, on a précisément critiqué Dewey pour avoir inspiré ce foisonnement et le mot d'ordre fut inverse : «Back to basics!», un mot d’ordre qui a horrifié les disciples de Dewey. Je ne suis pas sûr, cependant, que Dewey aurait été si opposé à ce mouvement, d'une part parce qu'il n’empêche pas que perdure un intérêt pour la diversité des activités, comme le montre notre questionnaire, d'autre part et surtout parce que les basics la langue, les sciences, les maths - sont trois disciplines « outils », utiles aux élèves quelle que soit leur vie ultérieure, trois disciplines dont deux au moins sont, on l'a vu, sont au cœur de l'éducation démocratique.

Une autre tendance actuelle jugée «anathème » par les disciples est l'accountability, ce mode de gouvernement de l'école (standards, évaluation périodique des élèves, mise en tutelle plus ou moins fortes des écoles où ils ne progressent pas assez) qui s’est répandu au cours des années 1990 dans plusieurs états américains, a été généralisé en 2002 à tout le territoire et qu’ Obama a décidé de conserver, alors même qu'il donne souvent l'impression que sa philosophie politique est proche de celle de Dewey (sa croyance dans les vertus du débat l'espace public, de l'expérimentation, des échanges en général ${ }^{9}$ ). Ce qui est critiqué par les disciples n'est pas l'idée que l'école doive rendre des comptes à la communauté, au contraire une caractéristique de l'école démocratique, c’est l’idée de norme : "Norms have little value to pluralist democrats » (Garrison et Neiman, 2003, p. 26). La critique est double. Les standards, soit le fait que tous les élèves doivent maîtriser les mêmes compétences dans les disciplines de base, contredirait l’idée que chacun doit développer sa propre singularité. Les tests contrediraient l'idée que la croissance est un processus continu, permanent. Mais il n'est pas sûr que l’on puisse aujourd’hui développer sa singularité sans une maîtrise minimale des trois outils concernés. Mais on voit mal en quoi mesurer la taille d'un enfant l'empêche de continuer à grandir. Ce que, en revanche, Dewey réclamerait en ce domaine est, me semble-til, que l'ensemble du processus ne soit pas confié aux experts. Le public doit participer à la définition de ce qui doit être enseigné à tous, les enseignants doivent participer à la définition du niveau qu'il est raisonnable d'espérer atteindre, la communauté scolaire doit participer à l'évaluation de son établissement. Il n'est pas sûr, après tout, que la politique scolaire d’Obama contredise sa philosophie politique.

\footnotetext{
${ }^{9}$ Exemple : « Le leadership américain ne consiste pas à dominer mais à former les conditions permettant à la communauté internationale d’agir de concert » (déclaration citée dans Le Monde du 20/03/2011).
} 
Hors des États-Unis, parmi les formes scolaires proches de l'école démocratique, on peut citer la pédagogie de projet, très utilisée dans le système scolaire québécois, efficace, équitable et accueillant aux élèves ${ }^{10}$, ou encore la définition des compétences mesurées dans l'enquête PISA : « Les items de sciences (...) demandent aux élèves d'identifier des questions d'ordre scientifique, d'expliquer des phénomènes de manière scientifique et d'utiliser des faits scientifiques (OCDE, 2007, p. 40) ; « La capacité mathématique est la capacité d’identifier et de comprendre le rôle que les mathématiques jouent dans le monde, d'émettre des jugements bien fondés, d'utiliser les mathématiques de façon conforme aux besoins d'un citoyen constructif, responsable et réfléchi »(OCDE, 2004, p. 37). Interpréter cette proximité n’est d'ailleurs pas facile, sauf à penser que Dewey a prévenu cent ans à l'avance les desiderata de l'OCDE, officine du grand capital comme on sait. Les concepteurs des épreuves de PISA, qui doivent commencer par élaborer un concept de ce qu'ils appelleront mathématiques ou sciences, ne sont pas des deweyens qui appliqueraient les théories de leur maître à penser. Ce sont des chercheurs qui mobilisent plutôt des travaux empiriques sur leur discipline et son enseignement. Le plus simple est donc de penser que Dewey avait une idée assez juste de l'éducation dans les sociétés modernes, qui fait que ceux qui construisent des épreuves adaptées aux besoins des élèves dans les sociétés démocratiques actuelles ne tombent pas très loin de ses conceptions.

En France, il faut distinguer le discours politique des politiques réellement conduites, ce qui signale d'ailleurs que le gouvernement de l'école pose problème dans ce pays. Depuis 2002, les politiques expliquent l'inefficacité de l'école par l'absence d'autorité des professeurs, par l'absence de respect des élèves, et cette décadence de l'école par celle de la société. Selon la loi de 2005 sur l'éducation, l'école a pour mission de «transmettre les connaissances » et de « faire partager les valeurs de la République ». Ce diagnostic comme ces objectifs sont très éloignés de l'école démocratique. En revanche, de nombreuses politiques éducatives récentes en sont proches, soit qu'elles visent à favoriser une communauté d'expériences (l'affaiblissement des filières dans la réforme du lycée), soit qu'elles ajoutent l'idée de compétences à celle de connaissances (le socle commun), soit qu'elles favorisent une approche plus expérimentale de l'enseignement des sciences (comme le font l'association « La main à la pâte » mais aussi les instructions officielles), soit encore qu'elles aillent dans le sens d'une individualisation de l'enseignement (les Programmes Personnalisés de Réussite

\footnotetext{
${ }^{10}$ Signalons aussi la « loi 88 » (2008), qui, dans ce même Québec, vise à « renforcer la démocratie scolaire » et à « garantir à la population une meilleure reddition de compte et une plus grande transparence ».
} 
Educative), soit enfin qu'elles favorisent la mobilisation des connaissances pour le traitement de questions réelles (les Travaux Personnels Encadrés). La dualité entre les discours politiques et les politiques scolaires s’observe aussi dans les représentations des enseignants. Interrogés sur les buts et les conditions de l'enseignement, ils se déclarent majoritairement en faveur de certains énoncés deweyens, en même temps qu’aucun ne donne de réponses résolument deweyennes, comme si le modèle deweyen avait contaminé le modèle durkheimien traditionnel en France, mais sans être capable de le remplacer. On note cependant que ceux qui ont une conception plus «deweyenne » des conditions actuelles de l'enseignement sont davantage favorables à l'ouverture de l'enseignement du second degré à la grande masse des élèves, et déclarent tirer une satisfaction un peu plus grande de leur métier (Meuret et Lambert, 2010).

La question principale quant à l'actualité de Dewey, cependant, est de savoir s’il peut être utile pour répondre aux défis que l'école affronte aujourd’hui. On peut discerner trois utilités. D’une part, il alerte sur l'importance, pour que l'école retrouve sa légitimité, d'arriver à un accord public sur les connaissances et compétences les mieux à mêmes de favoriser la diversité et la richesse des échanges. Prétendre, comme les ministres récents, arriver à cet accord en revenant au bon sens populaire contre les sophistications délétères des spécialistes est, nous avertit Dewey, une option populiste mais non démocratique. À cet égard, en France, une occasion semble avoir été perdue lors de la détermination du contenu du socle commun. En vérité, un tel débat devrait se nourrir de travaux empiriques encore balbutiants sur la nature des apprentissages qui sont le plus à même de favoriser l'intensité des échanges dans une société. D’autre part, l'école démocratique ferait sans doute à la langue française (écrite, orale) et aux langues étrangères une place plus importante qu'aujourd'hui, en particulier aux langues extra européennes. Elle proposerait peut être aussi aux élèves de choisir une option dans un éventail très vaste d'activités susceptibles d'alimenter une socialité non marchande (arts, jeux, etc.) à charge pour l'élève de faire preuve de persévérance et d’y acquérir une certaine excellence susceptible d'alimenter des échanges approfondis. Enfin, Dewey inviterait probablement à mettre au point des formes démocratiques de régulation de l'école, qui ne soient pas technocratiques et ne reposent pas non plus sur des mécanismes de marché, .

Dewey n'indique pas de solutions - on verra dans ces deux ouvrages que la traduction concrète de ses énoncés n’est pas le plus souvent évidente. Il donne une direction, une école au service de la richesse des échanges sociaux, et une méthode, l'expérimentation. Cela n’est pas rien. 
On a essayé ici de donner des raisons de lire aujourd'hui Démocratie et Éducation et Expérience et Éducation. Il reste à prévenir le lecteur que ce n’est pas une lecture facile. Non seulement le style de Dewey n'est pas très direct, mais aussi sa lecture est rendue complexe parce qu'il arrive qu’on croie lire ce qu’il pense alors qu’il est engagé dans la présentation d'une autre théorie, puis d'une autre encore, dont il cherchera ensuite à dépasser ce qui les oppose. Cette dialectique peut susciter un autre type d'incompréhension, celle de lire ces dépassements comme des compromis, comme le souci de mettre tout le monde d'accord. En vérité, il y a bien chez Dewey l'envie d'arriver à une position que tous puissent partager, parce qu'elle découle rationnellement d'une conception des buts et des conditions de l'enseignement sur laquelle tous peuvent en effet s'accorder. Nous savons aujourd'hui que cet accord est seulement le fait de ceux qui partagent sa passion pour une conception exigeante et ouverte de la démocratie.

Mars 2011

Bibliographie

BAudelot Ch. \& Establet R., L’Élitisme républicain, Paris, Le Seuil, 2009.

BuLle N., L’Imaginaire réformateur, Le Débat, mars-avril, 2010.

DEWEY J., « The reflex arc concept in psychology », Psychological Review, III, 1896.

Dewey J., « The psychology of effort », Philosophical Review VI, 1897 (Cet article et le precedent sont repris dans The Philosophy of John Dewey, J. McDermott, ed., Chicago, The University of Chicago Press, 1981).

DeweY J., 1899, The School and Society, in R.D. Archambault, ed., 1974, John Dewey on Education, Selected writings, Chicago, Chicago University Press.

DeWey J., Democracy and Education, Mineola (NY), Dover, 1916/2004. 
Dewey J., « Social Absolutism », in Boydson A. ed., 1983, Dewey J., The Middle Work, vol. 13, Carbondale, Southern Illinois University Press, 1921.

Dewey J., Le Public et ses problèmes, université de Pau et Léo Scheer, 1927/2003.

DewEy J., Experience and Education, New York, Touchstone, 1938/1997.

Festenstein M., Dewey’s political philosophy, Stanford Encyclopaedia of Philosophy, 2005 (en ligne).

Garrison J. \& Neiman A., « Pragmatism and Education », in The Blackwell Guide to the Philosophy of Education, N. Blake et al., eds., Maiden, Ma, Blackwell Publishing, 2003.

Gauchet M., Blais M.C., \& OtTavi D., Les Conditions de l'enseignement, Paris, Stock, 2008.

Gutmann A., Democratic Education, Princeton, NJ, Princeton University Press, 1999.

Hoy T., The Political Philosophy of John Dewey, Towards a Constructive Renewal, Westport, Co, Praeger, 1998.

LuRÇAT L., « Le pédagogisme facteur d’échec », Philosophie politique, n 10, Paris, PUF, 1999.

MenAnd L., The Metaphysical Club, New- York, Farrar, Strauss et Giroux, 2001.

Meuret D. \& LAmbert M., « Les buts et les conditions de l'enseignement selon les enseignants du second degré », Éducation et Didactique, 5(1), 2011.

Meuret D., Gouverner l'École, Paris, PUF, 2007.

RoRTY R., Conséquences du pragmatisme, Paris, Le Seuil, 1993.

SEMETSKy I., « The problematics of human activity, Gilles Deleuze and the deweyan legacy », Studies in Philosophy and Education, 22, 2003.

WeBER E.T., « Dewey and Rawls on Education », Human Studies, vol. 31, 2008.

ZASK J., « La politique comme expérimentation », introduction à Dewey J., Le Public et ses problèmes, 2003. 
OCDE, PISA 2006, Les Compétences en sciences, un atout pour réussir, vol. 1, Paris, OCDE, 2007.

OECD, Learning for tomorrow world, first results of PISA 2003, Paris, OCDE, 2004. 